\title{
Escolhas relacionadas ao uso de ajuda física aumentam afetos positivos após acidente vascular encefálico
}

http://dx.doi.org/10.11606/1807-5509201800020299

\author{
Shadia Mussa YUSSEF* \\ Suzete CHIVIACOWSKY*
}

*Escola Superior de Educação Física, Universidade Federal de Pelotas, Pelotas,

RS, Brasil.

\section{Resumo}

Este estudo observou os efeitos de escolhas relacionadas ao uso de ajuda física durante a marcha em adultos após acidente vascular encefálico (AVE). Dezoito adultos hemiparéticos (40 a 74 anos), após AVE, praticaram uma tarefa: andar uma distância de quatro metros ultrapassando obstáculos no menor tempo possivel, com a possibilidade de utilizar um apoio de membro superior (barra de apoio) sob duas diferentes condições de prática, autocontrolada ou externamente controlada. Os participantes praticaram 30 tentativas no primeiro dia, e um teste de retenção, consistindo de cinco tentativas sem uso de ajuda física, foi realizado no dia seguinte. Os resultados demonstraram melhora similar no tempo para completar o percurso em ambos os grupos. Os resultados de questionário mostraram maior nível de afetos positivos para os participantes do grupo autocontrolado em relação aos participantes do grupo externamente controlado. Conclui-se que o uso autocontrolado de ajuda física pode adicionar benefícios ao processo de melhora da marcha em sujeitos pós-AVE.

Palavras-Chave: Dano cerebral; Reabilitação; Marcha; Ajuda física; Autonomia; Afetos.

\section{Introdução}

O Acidente Vascular Encefálico (AVE) é uma doença cerebrovascular desencadeada por obstrução do fluxo sanguíneo para o encéfalo, através de rompimento de um vaso sanguíneo ou bloqueio por um coágulo, provocando déficits funcionais ao indivíduo ${ }^{1}$. Além da incapacidade funcional e custos com hospitalizaçóes, no Brasil o AVE ainda representa o principal motivo de morte em ambos os sexos ${ }^{2}$. A hemiparesia é o sinal mais evidente da doença, desencadeando uma postura assimétrica, com menor transferência de peso para o membro inferior parético, dificultando a marcha e a realização das atividades funcionais ${ }^{3}$.

Para os sujeitos acometidos por AVE a aprendizagem motora relaciona-se fundamentalmente ao processo de reaprender movimentos, através da prática, a fim de realizar suas necessidades de vida diária com sucesso, após a hemiparesia ${ }^{4}$. A capacidade de aprendizagem dos sujeitos pós AVE, no entanto, difere da aprendizagem de sujeitos típicos, sendo prejudicada por déficits cognitivos, sensitivos, perceptuais, de memória, de linguagem, além dos motores instalados após a lesão cerebral. Tais déficits normalmente resultam na redução da velocidade de processamento de informaçóes nestes sujeitos 5 . De forma geral, os pacientes que sofreram AVE recebem muitas informaçóes sobre o modo de realizar as tarefas de reaprendizagem e costumam exercer pouco controle sobre o ambiente de aprendizagem. Normalmente o que se observa é o controle total da sessão de prática pelos fisioterapeutas, podendo não ser esta uma condiçấo facilitadora da aprendizagem aos pacientes com AVE.

Uma variável interessante que vem sendo investigada recentemente na área da aprendizagem motora é a aprendizagem com autocontrole ${ }^{6}$. Esta se apresenta de forma diferente de abordagens anteriores relacionadas à aprendizagem motora, pois envolve condiçóes de prática em que o aprendiz detém algum controle sobre a mesma, sendo mais ativo durante o processo de aprendizado. Neste contexto, estudos sobre a prática autocontrolada mostram que maior 
aprendizagem é observada quando os participantes possuem autonomia sobre algum ou alguns dos fatores que compóem a prática ${ }^{7-11}$. A autonomia é considerada uma necessidade básica do ser humano, referindo-se especificamente à necessidade de controlar ou sentir-se agente de suas próprias açóes ao invés de sentir-se pressionado ou controlado ${ }^{12}$. Contextos de prática que suportem a autonomia do aprendiz são considerados potencialmente capazes de aumentar a motivação do mesmo ${ }^{8,13,14}$.

Mais especificamente em relação ao uso autocontrolado de assistência física, ressaltam-se os estudos de Hartman ${ }^{15}$ e Chiviacowsky, Wulf, LEWTHWAITE e CAMPos (2012) ${ }^{16}$, os quais testaram condições de prática autocontrolada, respectivamente em adultos típicos e com a doença de Parkinson, confirmando os benefícios deste tipo de prática em relação a condições de prática externamente controladas. Em ambos os estudos, os participantes que puderam escolher quando utilizar um bastão de equilíbrio enquanto se equilibravam em uma plataforma de equilíbrio, demonstraram maior aprendizagem da tarefa do que participantes sem chance de escolha. Pode-se considerar que o uso de assistência física se assemelha ao contexto do uso de órteses tipo muleta por sujeitos hemiparéticos por AVE, que apresentam o equilíbrio e a marcha prejudicados ${ }^{9,17,18}$. Sendo assim, os benefícios gerados pela prática autocontrolada, como a maior motivação e a possibilidade do aprendiz adequar a prática de acordo com as suas necessidades, ilustram a possibilidade e possíveis vantagens do uso deste arranjo de prática nas condiçôes de reabilitação com uso de assistência física, porém com cautela, visto que pesquisas com populaçóes especiais ainda são quase inexistentes ${ }^{7,9}$.

Em vista dos resultados positivos demonstrados nos estudos prévios sobre aprendizagem autocontrolada e a escassez de estudos investigando a aprendizagem motora em indivíduos com lesão no sistema nervoso central, considera-se importante a realização deste estudo. Devido às potenciais limitaçóes sensoriais, perceptivas e motoras (ex., déficits sensoriais, restrições articulares), principalmente associadas ao equilíbrio relacionado à marcha ${ }^{3}$, apresentadas por sujeitos pós AVE, considera-se importante investigar fatores de prática que possam otimizar a aprendizagem, facilitando a locomoção desta população. Ainda, estudos tem demonstrado que o fornecimento de escolhas pode aumentar o nível de afetos positivos dos aprendizes ${ }^{19}$. Desta forma, o objetivo do presente estudo foi examinar os efeitos das práticas com ou sem escolhas sobre o uso de ajuda física (barra de apoio), na aprendizagem da marcha ultrapassando obstáculos e nos estados afetivos de adultos hemiparéticos por AVE. Foi escolhida uma tarefa de equilíbrio dinâmico, com o intuito de trazer sua aplicabilidade ao cotidiano dessa população, a qual apresenta o equilíbrio deficitário pela diminuição da força muscular e informação sensorial no hemicorpo afetado, gerando assimetria postural ${ }^{20}$.

\section{Método}

\section{Participantes}

Participaram do estudo 18 adultos com quadro de hemiparesia ocasionada por AVE (12 homens e 6 mulheres), com grau de espasticidade entre 0 e 2 pela escala de Ashworth Modificada ${ }^{21}$, com média de idade de 57,8 anos (DP: 10,70. Os participantes não realizavam reabilitação no momento da pesquisa, não apresentavam déficits cognitivos (Mini Exame do estado mental) 22 ou visuais, outras doenças neurológicas associadas, instabilidade clínica ou deformidades em membros inferiores. Foram incluídos na amostra os participantes que apresentaram classificação leve de comprometimento motor, conforme domínio para membros inferiores da escala de FugL - Meyer ${ }^{23}$, variando entre 21 e 30 pontos. Ainda, todos os participantes apresentavam eficiência motora mínima no membro superior acometido, que permitisse a realizaçáo do apoio na barra. Os grupos foram equiparados por gênero e grau de espasticidade dos participantes, cujas características individuais são mostradas na TABELA 1 , além das idades serem próximas quando não era possível encontrar indivíduos com idade idêntica. Os participantes não possuíam conhecimento sobre o objetivo do experimento e assinaram o Termo de Consentimento Livre e Esclarecido, tendo o estudo sido aprovado pelo Comitê de Ética em Pesquisa da ESEF/UFPEL protocolo no 005/2011. 
TABELA 1 - Características dos participantes da amostra: MEEM (mini exame do estado mental); AM (escala de Ashworth modificada); FM-MMII (domínio de membros inferiores da escala de Fugl-Meyer).

\begin{tabular}{lcccccccccc}
\hline Sujeito & Idade & Sexo & Raça & $\begin{array}{c}\text { Último } \\
\text { AVE } \\
\text { meses }\end{array}$ & $\begin{array}{c}\text { Tipo de } \\
\text { AVE }\end{array}$ & MEEM & AM & $\begin{array}{c}\text { FM } \\
\text { MMII }\end{array}$ & $\begin{array}{c}\text { Hemi } \\
\text { paresia }\end{array}$ & $\begin{array}{c}\text { Fatores } \\
\text { de risco } \\
*\end{array}$ \\
\hline & & & & & AC & & & & & \\
\hline 1 & 60 & F & B & 11 & I & 22 & 1 & 25 & D & 3,5 \\
2 & 40 & F & B & 36 & H & 27 & 1 & 27 & E & 4 \\
3 & 74 & M & B & 60 & I & 29 & 1 & 30 & E & 1 \\
4 & 59 & M & B & 48 & I & 30 & 2 & 23 & D & $1,4,5$ \\
5 & 40 & F & B & 36 & H & 24 & $1+$ & 23 & D & $1,4,5$ \\
6 & 65 & M & B & 36 & I & 28 & $1+$ & 25 & D & $1,4,5$ \\
7 & 42 & M & B & 12 & I & 29 & 1 & 30 & E & 1 \\
8 & 60 & M & B & 12 & I & 29 & $1+$ & 28 & D & 1 \\
9 & 63 & M & B & 8 & I & 24 & 2 & 21 & E & 1,5 \\
\hline & & & & & EC & & & & & \\
\hline 10 & 61 & F & N & 24 & I & 29 & 1 & 24 & E & $1,3,4,5$ \\
11 & 72 & F & N & 26 & I & 24 & 1 & 21 & D & 1,5 \\
12 & 49 & M & N & 18 & I & 30 & 1 & 28 & D & 1,4 \\
13 & 54 & M & B & 24 & I & 25 & 2 & 25 & D & $1,2,5$ \\
14 & 63 & F & B & 84 & H & 26 & $1+$ & 27 & D & 1,3 \\
15 & 60 & M & B & 11 & H & 28 & $1+$ & 27 & E & 4,5 \\
16 & 45 & M & N & 7 & I & 29 & 1 & 23 & E & $1,3,5$ \\
17 & 61 & M & B & 11 & I & 28 & $1+$ & 26 & D & 3,4 \\
18 & 72 & M & N & 48 & I & 23 & 2 & 24 & E & 1 \\
\hline & & & & & & & & & &
\end{tabular}

\section{Equipamento e tarefa}

O equipamento utilizado consistiu em uma barra de apoio cilíndrica de madeira, posicionada a uma distância de $90 \mathrm{~cm}$ do chão, com quatro metros de comprimento. Dois obstáculos rígidos, medindo $57 \mathrm{~cm}$ de largura, $15 \mathrm{~cm}$ de comprimento e nove centímetros de altura, foram posicionados no percurso e simulavam obstáculos encontrados na vida diária dos participantes, permanecendo exatamente no centro da primeira e da segunda metade da barra de apoio. A tarefa consistia em andar o percurso de 4 metros ultrapassando os obstáculos no menor tempo possível, podendo utilizar ou não o apoio de membro superior, conforme instrução da experimentadora. O tempo de movimento (TM), ou seja, o tempo necessário para andar do início ao fim do percurso foi mensurado através de cronômetro (DLK, modelo WT038N/F). Cada tentativa iniciava com o participante posicionado atrás da linha de partida e terminava no momento em que o primeiro pé tocava o solo fora da área estipulada. A fim de verificar os efeitos da prática autocontrolada sobre os estados afetivos dos participantes, foi utilizado o Positive and Negative Affect Schedule Expanded $(\text { PANAS-X })^{24}$. O questionário foi preenchido, imediatamente após a fase de prática e após o teste de retenção, de acordo com uma escala de Likert de 1 a 7 , sendo 1 (nenhum pouco verdadeiro) e 7 (muito verdadeiro).

\section{Delineamento experimental e procedimentos}

Os 18 participantes foram alocados de forma aleatória para um dos dois grupos de prática: autocontrolado (AC), ou externamente controlado (EC). Ao grupo AC foi explicado que poderiam utilizar a assistência física oferecida pela barra de
*Fatores de risco:

1 - HAS; 2 - DM; 3

- Hipercolesterolemia;

4 - Tabagismo;

5- Inatividade física 
apoio, durante todo o percurso da tentativa, quando achassem necessário. Ao grupo EC foi informado que em algumas tentativas eles seriam avisados para usar o apoio da barra, e em outras não. Este grupo recebeu uma frequência de uso da assistência física de modo equiparado ao grupo AC, ou seja, nas mesmas tentativas que o sujeito correspondente do grupo AC utilizou o apoio. Os participantes foram informados que, uma vez escolhida (grupo AC) ou informada (grupo EC) a utilização da barra de apoio em uma determinada tentativa, esta deveria ser utilizada durante todo o percurso da tentativa. Todos os participantes receberam informaçôes de feedback ao final de cada tentativa, sendo estas relacionadas ao tempo utilizado para andar o percurso especificado. Foram também informados que realizariam uma prática sem o uso do apoio no dia seguinte. Os participantes realizaram a marcha no sentido que favorecesse a transferência de peso para o hemicorpo parético, o qual permanecia homolateral à barra de apoio para membro superior, na tentativa de tornar a tarefa funcional, com maior validade ecológica. Foram posicionados, assim, de acordo com o lado hemiparético (direito ou esquerdo), para definir o sentido do percurso que seria cronometrado. Todos os participantes realizaram, individualmente, 30 tentativas de prática, com 30 segundos de intervalo entre as tentativas. Com os participantes posicionados na linha de partida (início do percurso de 4 metros), o tempo de cada tentativa era mensurado tendo como início um sinal sonoro em conjunto com o acionamento do cronômetro e finalizava quando um dos pés tocava o solo após a linha final. $\mathrm{Na}$ fase de retenção, que ocorreu 24 horas após a fase de aquisição foram realizadas cinco tentativas da tarefa. Nesta fase experimental os participantes não puderam utilizar a assistência física. Imediatamente após o final da fase de prática e do teste de retenção, os participantes de ambos os grupos responderam ao questionário.

\section{Análise dos dados}

A variável dependente foi o TM utilizado para percorrer o percurso de 4 metros. Os dados foram analisados em blocos de tentativas. A normalidade dos dados e a homogeneidade de variância foram confirmadas pelos testes de Shapiro-Wilk e Levene. $\mathrm{Na}$ fase de prática, foi utilizada a análise de variância (ANOVA) two-way, 2 (grupos: AC versus EC) X 6 (blocos de 5 tentativas), com medidas repetidas no último fator. Os dados da fase de retenção foram analisados através da ANOVA one-way. O nível alpha de significância foi de 0,05 para todas as análises. Para analisar a comparaçáo entre as tentativas com e sem uso do apoio, foi utilizada uma ANOVA two-way, 2 (tipo de tentativa: com apoio versus sem apoio) X 2 (grupo: AC versus EC). Os resultados do questionário foram analisados através da ANOVA one-way. Os dados foram analisados através do programa estatístico SPSS versão 13.0.

\section{Resultados}

\section{Tempo de movimento}

\section{Prática}

$\mathrm{Na}$ análise dos dados (FIGURA 1, esquerda) foi observado que os dois grupos apresentaram diminuição nos TMs com o decorrer da prática.

Através da ANOVA pode-se observar que ambos os grupos modificaram o seu comportamento de forma significativa, diminuindo o TM através dos blocos de tentativas, $\mathrm{F}(5,80)=26,49, \mathrm{p}<$ $0,01, \mathrm{Eta}^{2}=.62$. Não foram observadas diferenças significativas entre os grupos durante a prática $\mathrm{F}(1,16)>1$; ou na interação entre blocos e grupos $F(5,80)<1$. Com relação a frequência de solicitação da assistência física (uso da barra de apoio) pelo grupo AC, foi observado um percentual médio de 33\%. Não foi encontrada diferença significativa em relação ao TM entre as tentativas com e sem uso da assistência física, em ambos os grupos, $\mathrm{F}(1,16)<1$, ou presença de interação entre estas variáveis, $\mathrm{F}(1,16)=1,10$, $\mathrm{p}>0,05$.

\section{Retenção}

Nesta fase, realizada 24 horas após a fase de prática, também não foi observada diferença significativa entre os grupos, $F(1,16)<1$ (FIGURA 1, direita). 
É possível observar, entretanto, uma tendência de interação entre o último bloco de tentativas da fase de prática e o bloco de retenção, com o grupo AC demonstrando uma melhora no seu
TM, enquanto o grupo EC demonstrou piora. A ANOVA demonstrou diferença marginalmente significante, $F(1,16)=3,06, p=0,09$ em relação a este aspecto.

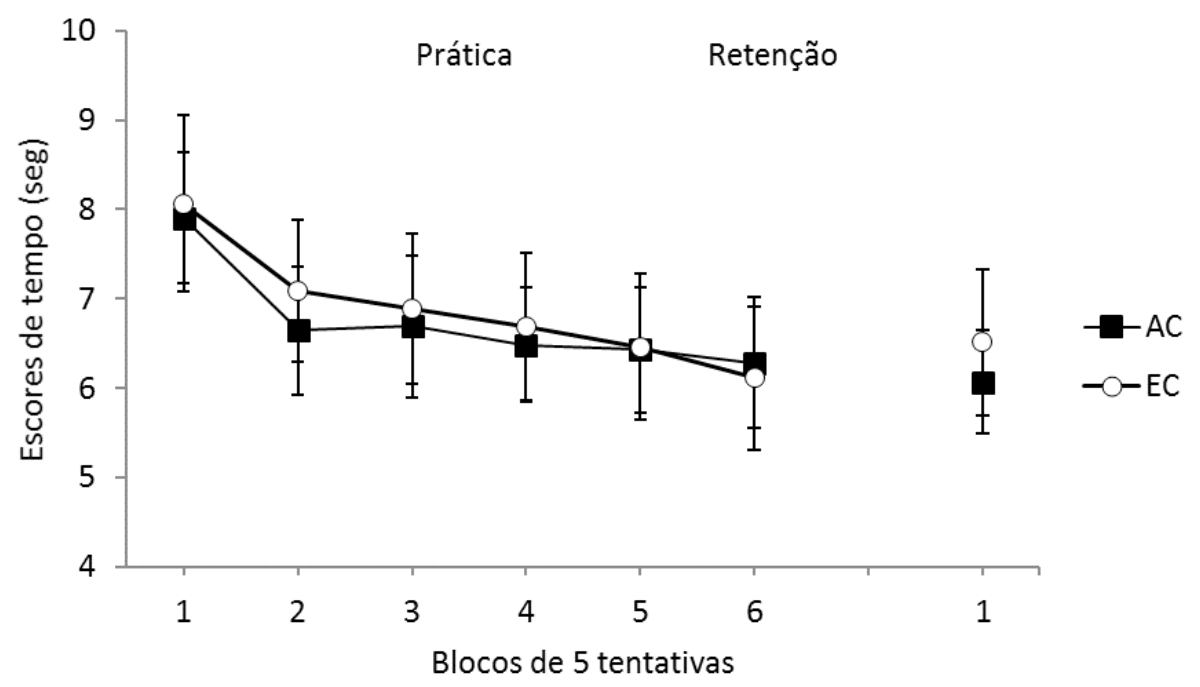

FIGURA 1 - Escores dos tempos de movimento dos grupos AC e EC, nas fases de prática e retenção. As barras de erros representam o erro padrão.

\section{Questionário}

$\mathrm{Na}$ análise do questionário PANAS - X, após a fase de prática, os resultados mostraram um maior grau de "interesse" na realização da tarefa para o grupo AC em relaçáo ao grupo $\mathrm{EC}, F(1,17)=$ $5,22, p<0,05, E t a^{2}=.24$, assim como uma diferença marginalmente significante em relação ao aspecto "calma", $F(1,17)=3,37, p=0,08, E t a^{2}=.17$ a favor do grupo AC. Quanto aos resultados do questionário aplicado após a retenção, foram observadas diferenças marginais, também favorecendo o grupo $\mathrm{AC}$ em relação aos seguintes itens: "alegre", $F(1,17)=4,11$, $p=0,06, E t a^{2}=.20$; “ousado", $F(1,17)=$ 3,69, $p=0,07, E t a^{2}=.18$; "forte", $F(1,17)=$ $3,66, p=0,07, E t a^{2}=.18$; "confiante", $F(1,17)=$ 3,57, $p=0,08, E t a^{2}=.18$; e "concentrado", $F(1,17)=3,55, p=0,08, E t a^{2}=.18$.

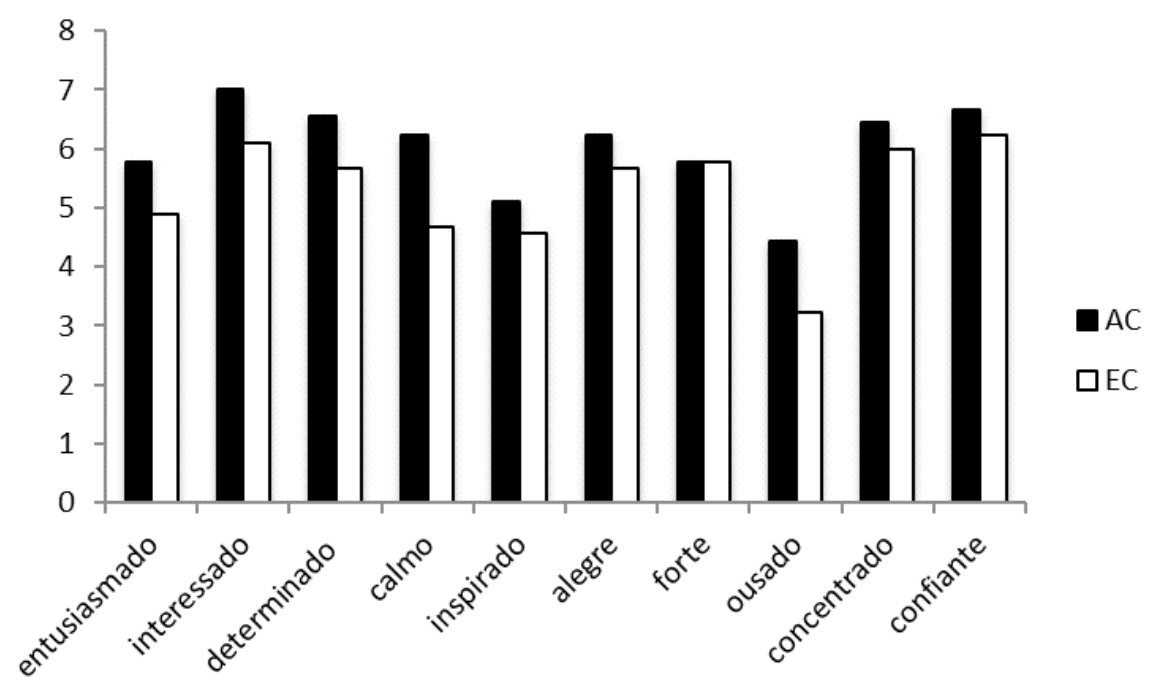

FIGURA 2 - Resultados do questionário PANAS dos grupos AC e EC, após a fase de prática. 


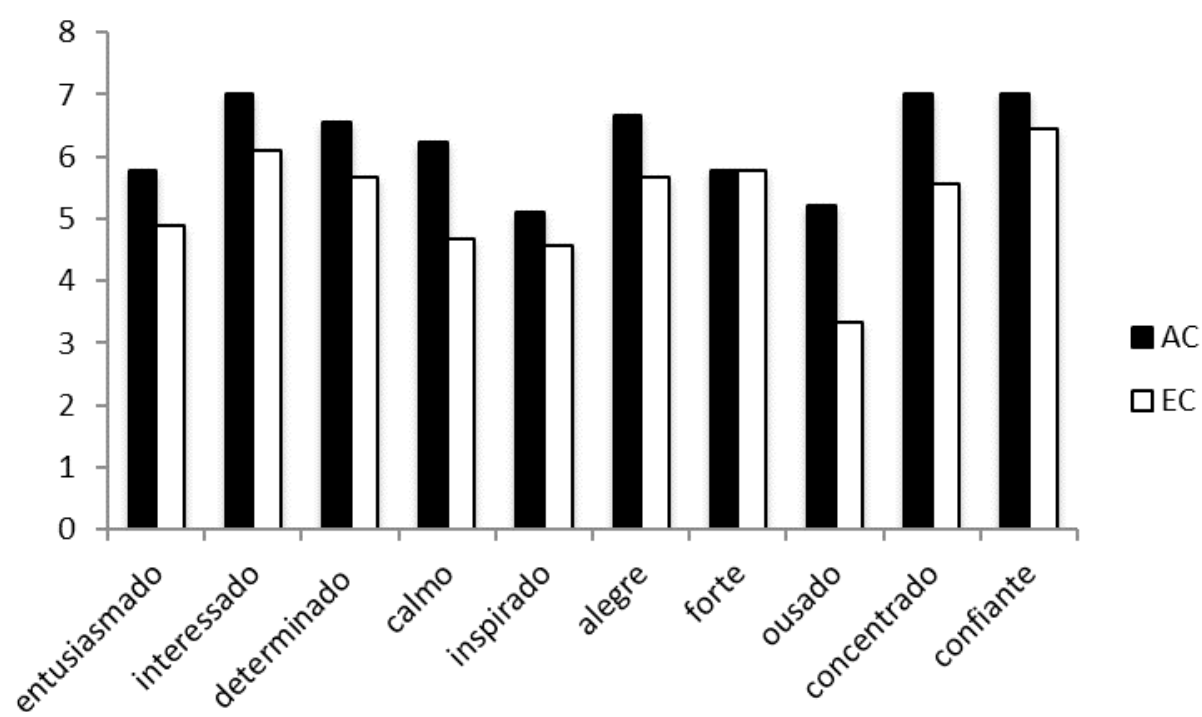

FIGURA 3 - Resultados do questionário PANAS dos grupos AC e EC, após a fase de retenção.

\section{Discussão}

Estudos têm demonstrado que o uso autocontrolado de assistência física pode beneficiar a aprendizagem motora $^{15,16}$. No entanto, ainda não eram conhecidos os efeitos deste tipo de prática na aprendizagem de tarefas motoras em sujeitos hemiparéticos por AVE. Os resultados do presente estudo demonstraram que participantes de ambos os grupos modificaram o seu comportamento na tarefa da marcha sobre obstáculos, demonstrando melhores TMs nos últimos blocos de tentativas da fase de prática e na fase de retençáo, quando comparados aos TMs iniciais. Tais achados indicam que mesmo em uma fase crônica, após lesão do sistema nervoso central, é possível que o indivíduo reaprenda uma tarefa motora, através do uso intermitente de ajuda física. Tal resultado está de acordo com estudo realizado por Boyd et al. ${ }^{25}$ envolvendo sujeitos na fase crônica do AVE, o qual, investigando as relaçóes entre o grau de severidade da doença e as tarefas aplicadas para avaliar a aprendizagem motora, verificou que a memória e a aprendizagem implícita não são completamente perdidas com o AVE.

Com relação a comparação entre os TMs nas tentativas com e sem o uso de assistência física oferecida pelo apoio para membro superior, os resultados indicaram a inexistência de diferença estatística significativa, para ambos os grupos. Tal resultado concorda com os resultados do estudo de HarTMANI ${ }^{5}$, no qual a diferença entre as tentativas com ou sem o uso do bastão não foi significativa. No entanto, o estudo difere dos resultados de CHIviacowsky et al. ${ }^{16}$, onde participantes com a doença de Parkinson beneficiaram-se do uso do bastáo, talvez por efeito de placebo. Especificamente em relação a sujeitos com AVE, BuUrke et al. ${ }^{26}$ demonstraram através de pesquisa envolvendo sujeitos hemiparéticos que deambulavam utilizando dispositivo auxiliar, que todos os participantes apresentavam velocidades relativamente mais baixas ao caminhar com o auxílio de dispositivo. Já o estudo de Tyson \& Rogerson ${ }^{27}$ encontrou melhora na mobilidade funcional e nos parâmetros espaço - temporais da marcha em pacientes pós AVE, ao caminhar com auxílio de dispositivos. Portanto, ainda existem controvérsias no campo da reabilitaçáo a respeito dos efeitos imediatos que a assistência física pode trazer à marcha de hemiparéticos. Mais interessante, no entanto, é o fato do presente estudo demonstrar melhora geral no desempenho da marcha com obstáculos através do uso intermitente de ajuda física durante a prática, e que esta melhora é relativamente permanente, como demonstram os resultados do teste de retenção realizados sem o uso de assistência física.

Com relaçáo à frequência de utilização da barra, verificou-se que os participantes do grupo autocontrolado solicitaram uma frequência média reduzida de utilização de assistência física de 33\%, valor semelhante às frequências solicitadas por sujeitos típicos durante a prática com autocontrole desta 
variável. Em um estudo prévio, os participantes solicitaram a utilização de assistência física, durante a tarefa de equilíbrio em estabilômetro, em 38\% das tentativas ${ }^{15}$. Frequência similar foi encontrada em experimento recente, com populaçấo de parkinsonianos $(41 \%)^{16}$. Tal resultado deve ser destacado, visto que o esperado seria que os sujeitos pós AVE solicitassem mais a assistência física autocontrolada, justamente por apresentarem comprometimento dos sistemas de feedback intrínseco ${ }^{28}$.

Com relação aos efeitos sobre a aprendizagem, os resultados do presente estudo indicam similaridade dos efeitos do arranjo autocontrolado de prática em relação ao arranjo externamente controlado em sujeitos hemiparéticos por AVE na aprendizagem da tarefa de equilíbrio, diferente de resultados de estudos prévios envolvendo arranjos de prática autocontrolada. No entanto, é importante ressaltar que o grupo AC demonstrou uma tendência de melhora no último bloco da fase de aquisição para o primeiro da retenção, enquanto o grupo EC demonstrou tendência de piora, com esta interaçáo entre os grupos apresentando diferença significativa marginal, o que pode ser considerado um fator favorável a este tipo de prática. Tal comportamento sugere que os participantes do grupo AC conseguiram manter a melhora do comportamento entre as diferentes fases da prática de forma mais eficiente do que os participantes do grupo EC.

Importante, os resultados demonstraram o efeito benéfico da prática autocontrolada sobre as dimensóes afetivas dos participantes durante a aquisição da tarefa, em relação à prática externamente controlada. Apesar deste efeito não ter contribuído diretamente aos resultados de desempenho e aprendizagem à curto prazo, é sabido que a motivação é um fator determinante no processo de reabilitaçáo, visto que a participação mais ativa dos sujeitos está relacionada ao melhor desempenho, e tratamentos que visam treinos mais passivos proporcionam menores graus de motivação ${ }^{29}$. Além dos benefícios motores que as práticas com maior caráter motivacional podem trazer aos sujeitos pós AVE, estas também podem auxiliar nos quadros depressivos que a maioria destes pacientes apresenta. Limitaçóes motoras nesta população nem sempre advém do déficit neurológico em si, mas de complicaçóes psiquiátricas, sendo a depressão a mais prevalente. Este quadro favorece a incapacitação do sujeito após o AVE, dificultando sua reabilitação e prolongando o tempo de recuperação ${ }^{30}$. Ao contrário, estados afetivos ligados, por exemplo, a um maior grau de interesse pela tarefa podem levar a um maior engajamento dos participantes em sessōes de reabilitação, com consequências benéficas à aprendizagem a longo prazo.

O conjunto dos resultados alcançados permite inferir, assim como demonstrado por estudo anterior $^{31}$, a importância da autonomia para o processo de aprendizagem motora de sujeitos hemiparéticos pós AVE. Porém, evidencia um campo onde é preciso haver mais investigaçóes que relacionem os fatores que afetam a aprendizagem motora e a reabilitaçáo. A prática autocontrolada possui o potencial de beneficiar o processo de re-aprendizagem ou "conquistas motoras" dos sujeitos em fase crônica do AVE, trazendo maior interesse e potencial engajamento. Futuros estudos poderiam ser realizados com outros tipos de tarefa e variáveis a serem controladas pelos aprendizes, como por exemplo a demonstração e o feedback autocontrolado. Ainda, o presente estudo limitou-se a avaliar o tempo de movimento com variável dependente. Sugere-se a realização de experimentos utilizando também a análise do padrão de movimento da marcha.

\section{Conflito de interesse}

Os autores não apresentam nenhum potencial conflito de interesse em relação ao presente manuscrito.

\section{Abstract}

\section{Choices over physical aid enhance positive affect after stroke}

The present study looked at the effects of choices over physical aid during gait in adults after stroke. Eighteen adults post-stroke from unilateral cerebrovascular accident (40-74 years) practiced walking a distance of $4 \mathrm{~m}$, overcoming 2 obstacles in the shortest time possible, with the possibility of using physical 
aid (support bar) under one of two practice conditions: self-controlled or externally controlled. Participants practiced 30 trials in the first day, and a retention test was performed one day later consisting of five trials without the use of physical aid. The results showed a similar significant improvement in time to complete the task for the groups. Questionnaire results showed that the self-controlled group demonstrated higher levels of positive affect compared with the externally controlled group. The findings show that providing participants with choices about the use of physical aid add benefits to the learning process of stepping over obstacles after stroke.

KEYwORDS: Brain damage; Recovery; Gait; Physical aid; Autonomy; Affects.

\section{Referências}

1. Rosamond W, Flegal K, Furie K, Go A, Greenlund K, Haase N, et al. Heart disease and stroke statistics: 2008 update - a report from the American Heart Association Statistics Committee and Stroke Statistics Subcommittee. Circulation. 2008;117(4):e25-e146.

2. Ministério da Saúde (BR). Saúde lança consulta pública para o aprimoramento da assistência a pacientes com AVC [Internet]. Brasília; 2010 [citado em 2015 Fev 5]. Disponível em: http://www.brasil.gov.br/noticias/saude/2010/10/ saude-abre-consulta-publica-sobre-assistencia-a-pacientes-com-avc.

3. Van Peepen RPS, Hendriks HJM, van Meeteren NLU, Helders PJM, Kwakell G. The development of a clinical practice stroke guideline for physiotherapists in the Netherlands: a systematic review of available evidence. Disabil Rehabil. 2007;29(10):767-83.

4. Van Vliet PM, Wulf G. Extrinsic feedback for motor learning after stroke: what is the evidence? Disabil Rehabil. 2006;28(13-14):831-40.

5. Vidoni ED, Boyd LA. Preserved motor learning after stroke is related to the degree of proprioceptive deficit. Behav Brain Funct. 2009;5:36.

6. Sanli EA, Patterson JT, Bray SR, Lee TD. Understanding self-controlled motor learning protocols through the selfdetermination theory. Front Psychol. 2013;3:611.

7. Chiviacowsky S, Wulf G. Self-controlled feedback: does it enhance learning because performers get feedback when they need it? Res Q Exerc Sport. 2002;73(4):408-15.

8. Chiviacowsky S. Self-controlled practice: autonomy protects perceptions of competence and enhances motor learning. Psychol Sport Exerc. 2014;15(5):505-10.

9. Wulf G. Self-controlled practice enhances motor learning: implications for physiotherapy. Physiotherapy. 2007;93(2):96-101.

10. Chiviacowsky S, Wulf G, Medeiros FL, Kaefer A. Learning benefits of self-controlled knowledge of results in 10-year old children. Res Q Exerc Sport. 2008;79(3):405-10.

11. Patterson JT, Carter M. Learner regulated knowledge of results during the acquisition of multiple timing goals. Hum Mov Sci. 2010;29(2):214-27.

12. Ryan RM. Psychological needs and the facilitation of integrative processes. J Pers. 1995;63(3):397-427.

13. Deci EL, Ryan RM. The "what" and "why" of goal pursuits: human needs and the self-determination of behavior. Psychol Inq. 2000;11(4):227-68.

14. Lewthwaite R, Chiviacowsky S, Drews R, Wulf, G. Choose to move: the motivacional impact of autonomy support on motor learning. Psychon Bull Rev. 2015;22(5):1383-8.

15. Hartman JM. Self-controlled use of a perceived physical assistance device during a balancing task. Percept Mot Skills. 2007;104(3 Pt. 1):1005-16.

16. Chiviacowsky S, Wulf G, Lewthwaite R, Campos T. Motor learning benefits of self-controlled practice in persons with Parkinson's disease. Gait Posture. 2012;35(4):601-5.

17. Van Peppen RP, Kwakkel G, Wood-Dauphinee S, Hendriks HJ, van der Wees PJ, Dekker J. The impact of physical therapy on functional outcomes after stroke: what's the evidence? Clin Rehabil. 2004;18(8):833-62.

18. Bateni H, Maki BE. Assistive devices for balance and mobility: benefits, demands, and adverse consequences. Arch Phys Med Rehabil. 2005;86(1):134-45. 
19. Hooyman A, Wulf G, Lewthwaite R. Impacts of autonomy-supportive versus controlling instructional language on motor learning. Hum Mov Sci. 2014;36:190-98.

20. Leroux A, Pinet H, Nadeau S. Task-oriented intervention in chronic stroke. Am J Phys Med Rehabil. 2006;85(10):820-30.

21. Bohhanon RW, Smith MB. Interrater reliability of a modified Ashworth scale of muscle spasticity. Phys Ther. 1987;67(2):206-7.

22. Brucki SMD, Nitrini CP, Bertolucii PHF, Okamoto IH. Sugestôes para o uso do Mini-Exame Mental no Brasil. Arqu Neuropsiquiatr. 2003;61(3-8):777-81.

23. Gladstone DJ, Danells CJ, Black SE. The Fugl-Meyer assessment of motor recovery after stroke: a critical review of its measurement properties. Neurorehabil Neural Repair. 2002;16(3):232-40.

24. Watson D, Clark LA. The PANAS-X: manual for the positive and negative affect schedule: expanded form. Psychol. 1994;52:409-18.

25. Boyd LA, Quaney BM, Pohl PS, Winstein CJ. Learning implicitly: effects of task and severity after stroke. Neurorehabil Neural Repair. 2007;21(5):444-54.

26. Buurke JH, Hermens HJ, Erren-Wolters CV, Nene AV. The effect of walking aids on muscle activation patterns during walking in stroke patients. Gait Posture. 2005;22(2):164-70.

27. Tyson SF, Rogerson L. Assistive walking devices in nonambulant patients undergoing rehabilitation after stroke: the effects of the functional mobility, walking impairments, and patients' opinion. Arch Phys Med Rehabil. 2009;90(3):475-79.

28. Robertson JVG, Hoellinger T, Lindberg P, Bensmail D, Hanneton S, Brami AR. Effect of auditory feedback differs according to side of hemiparesis: a comparative pilot study. J Neuroeng Rehabil. 2009;6:45.

29. Maclean N, Pound P, Wolfe C, Rudd A. The concept of patient motivation: a quantitative analysis of stroke professionals attitudes. Stroke. 2002;33(2):444-48.

30. Bilge C, Coçer E, Coçer A, Börü UT. Depression of functional outcome after stroke: the effect of antidepressant therapy on functional recovery. Eur J Phys Rehab Med. 2008;44(1):13-8.

31. Colombo R, Pisano F, Mazzone A, et al. Design strategies to improve patient motivation during robot-aided rehabilitation. J Neuroeng Rehabil. 2007;4:3.

\begin{tabular}{|c|c|}
\hline $\begin{array}{r}\text { ENDEREÇO } \\
\text { Suzete Chiviacowsky } \\
\text { Rua Luís de Camões, 625 } \\
\text { 96055-630 - Pelotas - RS - BRASIL } \\
\text { e-mail: suzete@ufpel.edu.br }\end{array}$ & 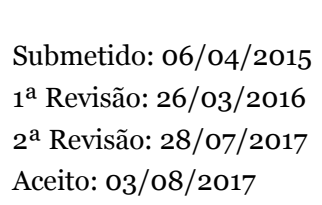 \\
\hline
\end{tabular}


